\title{
A Practical Physical-Layer Network Coding for Fading Channels
}

\author{
Bang Chul Jung, KIMICS, Member
}

\begin{abstract}
In the conventional PNC scheme, the relay node requires simultaneous transmission of two source nodes with strict power control and carrier-phase matching between two received symbols. However, this preequalization process at source nodes is not practical in fading channels. In this letter, we propose a novel physicallayer network coding (PNC) scheme with log-likelihood ratio (LLR) conversion for fading channels, which utilizes not pre-equalizer at transmitters (source nodes) but joint detector at receiver (relay node). The proposed PNC requires only channel side information at the receiver (CSIR), which is far more practical assumption in fading channels. In addition, the proposed PNC scheme can use the conventional modulation scheme like $M$-QAM regardless of modulation order, while the conventional PNC scheme requires reconfiguration of modulation scheme at the source nodes for detection of the received signal at relay node. We consider the combination of the proposed PNC and channel coding, and find that the proposed PNC scheme is easily combined the linear channel codes such as turbo codes, LDPC, and convolutional codes.
\end{abstract}

Index Terms - Two-way relaying technique, physicallayer network coding, channel coding, fading channels

\section{INTRODUCTION}

Future wireless communication networks are required to support increased high data rates, extended coverage, low latency, low deployment cost, and so on [1]. For satisfying theses requirements, most wireless communication systems have been evolved [2]-[4]. Relaying technique has been considered as a promising solution for providing extended coverage with low deployment cost [5]. However, the conventional halfduplex relaying technique causes a substantial loss in spectral efficiency because the transmission of one information symbol from the source node to destination node occupies two channel uses [6]. One way to avoid this spectral waste is to use a full-duplex relay that can transmit and receive simultaneously but the full-duplex relay is not easy to implement [7].

\footnotetext{
Manuscript received October 27, 2010; revised November 2, 2010; accepted November 11, 2010

Bang Chul Jung is with the Dept. of Information and Communication Engineering and the Institute of Marine Industry, Collage of Marine Science, Gyeongsang National University, Tongyeong, Gyeongnam, 650-160, Korea (Email: bcjung@gnu.ac.kr)
}

Network coding (NC) has emerged as a promising technique since its introduction [8] and it employs intermediate nodes to combine and code packets, while the conventional coding approaches code individual packets. NC was originally proposed in wired communication networks but there has been applied to wireless communication scenarios. In fact, it is reasonable to apply $\mathrm{NC}$ to wireless communications due to the broadcast nature of wireless medium. Two-way relaying technique based on $\mathrm{NC}$ utilizes resource more effectively compared to the conventional half duplex relaying and thereby dramatically improves the achievable rate of relay systems [9], [10]. With standard $\mathrm{NC}$, each source node utilizes one time-slot to transmit a packet to the relay and the relay takes the exclusive-or (XOR) of these two packet and broadcasts the XOR-ed packet during the third time-slot. Hence, the NC-based relaying technique uses only three time-slots, while the conventional half-duplex relaying technique uses four time-slots [11].

Physical-layer network coding (PNC) technique was proposed as a more efficient data relaying scheme, which enables two source nodes to transmit packets at the same time during a single time-slot [12]. The relay directly decodes the XOR-ed packet of the received packets rather than individual packets from source nodes and it broadcasts the decoded packet during the second time-slot. Hence, PNC requires only two time-slots for data exchange between two source nodes. In the original paper, the authors assumed that the wireless channel between each transmitter and receiver is modeled as additive white Gaussian noise (AWGN) channel through perfect power control and accurate phase compensation (which is also called pre-equalization) at the source nodes during the first time-slot. However, ideal channel estimation is required for pre-equalization and the preequalization with channel inversion is known to be quite power-inefficient in wireless communication links [13]. Therefore, this pre-equalization technique is not practical to implement in wireless communication scenarios with large scale path-loss and multi-path fading. Furthermore, the authors of [12] only considered uncoded packets even though channel coding techniques are used for error correction in the practical wireless communications.

There have been several studies on PNC technique for fading channels. In [14], the authors indicated that the conventional PNC technique [12] cannot be directly 
applied to the practical wireless systems and they provided a novel PNC technique which does not require channel information at transmitters. The author utilized the multiple relay selection scheme for improving the throughput performance but they only considered amplify-and-forward (AF) strategy due to the difficulty of extension of decode-and-forward (DF) based PNC to fading environments. In [15], [16], the denoise-andforward (DNF) strategy, which is a novel PNC technique in fading channels, was proposed. The key idea of DNF strategy comes from the fact that the relay node does not need to decode the both packets from the source nodes. In DNF scheme, the relay node maps the received packet to a discrete set of codewords and it broadcasts the codeword to which the received is mapped. The mapping process is referred to as denoising. However, this denoising process was not clearly explained and the authors only provided the criteria that a denoising mapping should satisfy but no algorithm to dynamically generate the mapping.

In this letter, we provide a practical PNC technique which is appropriate wireless communication systems where the fading effect exists. The proposed technique does not require pre-equalization at transmitters and utilizes a well-known joint maximal-likelihood (ML) detection based on log-likelihood ratio (LLR) for decoding the superposed signal received at relay node from both transmitters. We also consider the joint design of the proposed PNC and channel coding. The rest of this letter is organized as follows: In Section II, we propose a novel PNC scheme with a joint-ML detector and consider two types of channel decoding schemes in proposed PNC. In Section III, some numerical examples are shown. Finally, conclusions are drawn in Section IV.

\section{PRACTICAL PHYSICAL LAYER NETWORK CODING}

\section{A. System model}

We consider the three-node linear network model which consists of two source nodes $(A$ and $C$ ) and a single relay node $(B)$ between source nodes in Fig. 1. At time slot $1, A$ and $C$ transmit data simultaneously, and $B$ receives the superposed signal of $A$ and $C$. The relay node $B$ decodes the received signal in time slot 1 and produces a network-coded packet. We assume that the relay node use the exclusive OR (XOR) operation as a network coding scheme in this letter. Hence, at time slot $2, B$ broadcasts the network-coded packet with XOR operation of the source nodes if the decoding is succeeded. After receiving and decoding the packet from $B$ at time slot 2, the source nodes ( $A$ and $C$ ) perform XOR operation for the received packet and its own transmitted data at time slot 1. Then, both $A$ and $C$ can obtain the packets for themselves.
In general wireless communication systems, information bits (b) are protected by channel coding technique such as convolutional codes, turbo codes, and LDPC codes. After the channel coding process the coded bits (c) are modulated for transmission. The modulated signal is denoted by $\mathrm{x}$ and XOR operator is represented by $\oplus$.

Hence, the $l$-th received signal at relay node at time slot 1 is expressed as:

$$
y_{B}(l)=h_{A B} x_{A}(l)+h_{C B} x_{C}(l)+n_{B}(l),
$$

where $h_{A B}$ and $h_{C B}$ represent the wireless channel coefficients between the source nodes $(A$ and $C$ ) and the relay node (B), respectively. The term $n(l)$ indicates the additive white Gaussian noise at

$B\left(n(l) \sim C N\left(0, \sigma_{B}^{2}\right)\right)$. The wireless channel is assumed to be Rayleigh distributed, i.e., $h_{i j} \sim C N\left(0, \sigma_{i j}^{2}\right)$. The relay node is assumed to know the channel coefficients.

Fig. 2 shows a received signal at relay node at time slot 1 and we assume that both source nodes use BPSK modulation in this figure. Hence, there exist four signal candidates $\tilde{y}$ according to transmitted signal combination, i.e., $\left(x_{A B}, x_{C B}\right) \in\{(1,1),(1,-1),(-1,1),(-1,-1)\}$.

The $l$-th symbol received at $\mathrm{A}$ and $\mathrm{C}$ at time slot 2 , respectively, can be expressed as:

$$
\begin{gathered}
y_{A}(l)=h_{A B} x_{B}(l)+n_{A}(l) \\
y_{C}(l)=h_{C B} x_{B}(l)+n_{C}(l),
\end{gathered}
$$

where $x_{B}(l)$ represents the modulated symbol of network-coded packet from $B$. We assume that $\sigma_{A}^{2}=\sigma_{B}^{2}=\sigma_{C}^{2}$.
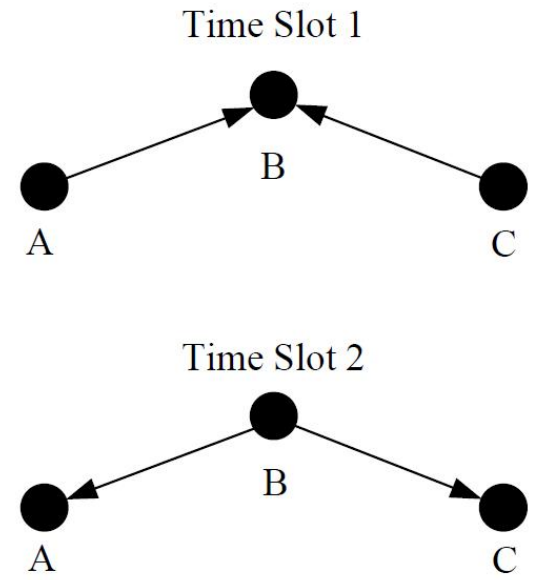

Fig. 1. System model 


\section{B. Joint detection at time slot 1}

In fact, it is the main challenging issue to obtain the network-coded packet from the superposed signal at relay node at time slot 1 in PNC technique. Once the networkcoded packet, i.e., $b_{B} \triangleq b_{A} \oplus B_{C}$, is obtained at the relay node, the packet is encoded, modulated, and broadcasted at time slot 2 . The decoding of networkcoded packet is the same operation as that in conventional communication systems and the performance depends on the channel state of each link. However, signal detection and channel decoding for the superposed signal at relay node at time slot 1 is not trivial and the decoding performance of the network-coded packet depends on both channel states of $A$ and $C\left(h_{A B}\right.$ and $\left.h_{C B}\right)$. To know the decoding performance of the network-coded packet at relay node is important because each source node uses it for transmit rate selection or modulation and coding scheme (MCS) selection. Therefore, we focus on how to obtain the network-coded packet $\left(b_{B}\right)$ from Eq. (1) in this letter.

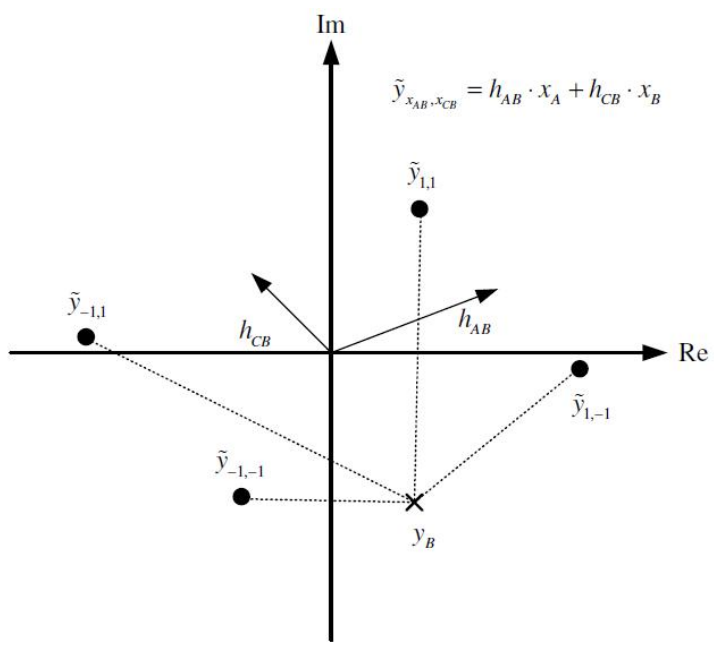

Fig. 2. Received signal at relay node at time slot 1 when both sources transmit BPSK symbols

\section{Separated decoding vs Direct decoding}

For decoding $b_{B}$, two decoding strategy can be used (separated decoding and direct decoding). In separate decoding, both $x_{A}$ and $x_{C}$ are decoded from Eq. (1) by using joint maximum likelihood (ML) detection with log-likelihood ratio (LLR) computation. This scheme needs to decode the received packet twice. After decoding the both packets, the network-coded packet is obtained by XOR operation between two decoded packets. As illustrated in Fig. 2, the received signal $Y_{B}$ is supposed to be equal to one of the four signal candidates if noise is not exist. The signal candidates are known to the relay node since we assume that the channel states and the modulation schemes used at transmitters are known to relay node. In separate decoding, the log-likelihood ratio
(LLR) value of both $c_{A}$ and $c_{C}$ can be obtained by using following equation.

$$
\begin{aligned}
& \Lambda\left(c_{A}\right)=\log \frac{\operatorname{Pr}\left(c_{A}=0 \mid y_{B}\right)}{\operatorname{Pr}\left(c_{A}=1 \mid y_{B}\right)}, \\
& \Lambda\left(c_{C}\right)=\log \frac{\operatorname{Pr}\left(c_{C}=0 \mid y_{B}\right)}{\operatorname{Pr}\left(c_{C}=1 \mid y_{B}\right)},
\end{aligned}
$$

where $\operatorname{Pr}(\Omega)$ is the probability that the event $\Omega$ occurs. Using symbol mapping rule of the modulation scheme, the probability in Eqs. (4) and (5) can be computed. For example, if both source nodes use BPSK modulation with mapping rule that $0 \rightarrow 1$ and $1 \rightarrow-1$, then Eqs. (4) and (5) can be rewritten as:

$$
\begin{aligned}
\Lambda\left(c_{A}\right)= & \log \frac{\operatorname{Pr}\left(y_{B} \mid x_{A}=1, x_{C}=1\right)+\operatorname{Pr}\left(y_{B} \mid x_{A}=1, x_{C}=-1\right)}{\operatorname{Pr}\left(y_{B} \mid x_{A}=-1, x_{C}=1\right)+\operatorname{Pr}\left(y_{B} \mid x_{A}=-1, x_{C}=1\right)} \\
& =\log \frac{\exp \left(-\frac{\left|y_{\mathrm{B}}-\tilde{y}_{1,1}\right|^{2}}{2 \sigma^{2}}\right)+\exp \left(-\frac{\left|y_{\mathrm{B}}-\tilde{y}_{1,-1}\right|^{2}}{2 \sigma^{2}}\right)}{\exp \left(-\frac{\left|y_{\mathrm{B}}-\tilde{y}_{-1,1}\right|^{2}}{2 \sigma^{2}}\right)+\exp \left(-\frac{\left|y_{\mathrm{B}}-\tilde{y}_{-1,-1}\right|^{2}}{2 \sigma^{2}}\right)}, \\
\Lambda\left(c_{C}\right) & =\log \frac{\operatorname{Pr}\left(y_{B} \mid x_{A}=1, x_{C}=1\right)+\operatorname{Pr}\left(y_{B} \mid x_{A}=-1, x_{C}=1\right)}{\operatorname{Pr}\left(y_{B} \mid x_{A}=1, x_{C}=-1\right)+\operatorname{Pr}\left(y_{B} \mid x_{A}=-1, x_{C}=-1\right)} \\
& =\log \frac{\exp \left(-\frac{\left|y_{\mathrm{B}}-\tilde{y}_{1,1}\right|^{2}}{2 \sigma^{2}}\right)+\exp \left(-\frac{\left|y_{\mathrm{B}}-\tilde{y}_{-1,1}\right|^{2}}{2 \sigma^{2}}\right)}{\exp \left(-\frac{\left|y_{\mathrm{B}}-\tilde{y}_{1,-1}\right|^{2}}{2 \sigma^{2}}\right)+\exp \left(-\frac{\left|y_{\mathrm{B}}-\tilde{y}_{-1,-1}\right|^{2}}{2 \sigma^{2}}\right)},
\end{aligned}
$$

where we assume the modulation symbols are generated with equal probability and use Bayes' rule. For high-order modulation schemes like 16-QAM, the LLR computation is also possible with increased complexity. The number of signal candidates exponentially increases as the modulation order at source nodes increases. Let the source nodes use M-QAM. Then, the number of signal candidates at relay node is equal to $M^{2}$. Each LLR value enters the channel decoder and we can obtain both information bits $b_{A}$ and $b_{C}$ if decoding errors are not exist. Thus, in separate decoding, two decoding processes are required. The network-coding are performed; $b_{B}=b_{A} \oplus B_{C}$.

On the other hand, most power in baseband signal processing is consumed by the channel decoder and the relay node needs to reduce its power for packet decoding. We propose a direct decoding scheme for improving the power efficiency of the relay node. The basic idea of the direct decoding is to use the linear property of the general channel codes, i.e., the linear combination of the two codewords becomes another codeword which is generated from exactly the same coding scheme with the same length. Most practical 
channel codes, which include convolutional codes, turbo codes, and LDPC codes, are linear codes. By applying this property of channel codes and the fact that the XOR operation is also linear mapping, it is easily proved that the order of channel coding and network coding can be exchanged. The linear property of channel codes can be written as:

$$
\begin{aligned}
c_{A} & =\Phi\left(b_{A}\right), \\
c_{C} & =\Phi\left(b_{C}\right), \\
c_{A} \oplus c_{C} & =\Phi\left(b_{A}\right) \oplus \Phi\left(b_{C}\right)=\Phi\left(b_{A} \oplus b_{C}\right) \triangleq \Phi\left(b_{B}\right),
\end{aligned}
$$

where $\Phi(\cdot)$ indicates the encoding function and the corresponding decoding function is denoted by $\Phi^{-1}(\cdot)$. Hence, as for the special case that there is no decoding error, the following relation is also valid.

$$
\begin{aligned}
& b_{A}=\Phi^{-1}\left(c_{A}\right), \\
& b_{C}=\Phi^{-1}\left(c_{C}\right), \\
& b_{B} \triangleq b_{A} \oplus b_{C}=\Phi^{-1}\left(c_{A}\right) \oplus \Phi^{-1}\left(c_{C}\right)=\Phi^{-1}\left(c_{A} \oplus c_{C}\right) .
\end{aligned}
$$

From Eq. (9), we find that the network-coded packet $b_{B}$ can be obtained through a single decoding if we directly compute the LLR-value of $\Lambda\left(c_{A} \oplus c_{C}\right)$ from the received signal at relay node $B$. We can compute $\Lambda\left(c_{A} \oplus c_{C}\right)$ by re-arranging terms in Eq. (6) or Eq. (7).

In direct decoding, the log-likelihood ratio (LLR) value of $c_{A} \oplus c_{C}$ can be obtained as:

$$
\Lambda\left(c_{A} \oplus c_{C}\right)=\log \frac{\operatorname{Pr}\left(c_{A} \oplus c_{C}=0 \mid y_{B}\right)}{\operatorname{Pr}\left(c_{A} \oplus c_{C}=1 \mid y_{B}\right)} .
$$

When we assume that BPSK modulation is used in both source nodes, the the log-likelihood ratio (LLR) value of $c_{A} \oplus c_{C}$ expressed as

$$
\begin{gathered}
\Lambda\left(c_{A} \oplus c_{c}\right)=\log \frac{\operatorname{Pr}\left(y_{B} \mid x_{A}=1, x_{C}=1\right)+\operatorname{Pr}\left(y_{B} \mid x_{A}=-1, x_{C}=-1\right)}{\operatorname{Pr}\left(y_{B} \mid x_{A}=-1, x_{C}=1\right)+\operatorname{Pr}\left(y_{B} \mid x_{A}=-1, x_{C}=1\right)} \\
=\log \frac{\exp \left(-\frac{\left|y_{\mathrm{B}}-\tilde{y}_{1,1}\right|^{2}}{2 \sigma^{2}}\right)+\exp \left(-\frac{\left|y_{\mathrm{B}}-\tilde{y}_{1,-1}\right|^{2}}{2 \sigma^{2}}\right)}{\exp \left(-\frac{\left|\tilde{y}_{\mathrm{B}}-\tilde{y}_{-1,1}\right|^{2}}{2 \sigma^{2}}\right)+\exp \left(-\frac{\left|y_{\mathrm{B}}-\tilde{y}_{-1,-1}\right|^{2}}{2 \sigma^{2}}\right)} .
\end{gathered}
$$

The computed LLR value enters the channel decoder and we can directly obtain the network coded packet $b_{B}$ otherwise decoding errors exist. Thus, in direct decoding, a single decoding process is required.

\section{NUMERICAL EXAMPLES}

Fig. 3 shows the uncoded bit-error rate (BER) performance of the proposed PNC technique at relay node $B$ at time slot 1 for varying $\left(E_{b} / N_{0}\right)$ and varying $\rho$, where $\rho$ is defined as $\sigma_{C B}^{2} / \sigma_{A B}^{2}$ and indicates the ratio of the average received signal-to-noise ratio (SNR) at relay node between two links. We change the $\left(E_{b} / N_{0}\right)$ of the link between $A$ and B $\left(\left(E_{b} / N_{0}\right)_{A B}\right)$ in this figure but the performance for varying the $\left(E_{b} / N_{0}\right)$ of the link between $C$ and $B$ is also expected by changing $\rho$. As we noted before, the BER performance of the PNC technique depends on both links. For example, when $\rho=1$, the approximate twice energy is required for the same BER performance, compared to the case that conventional BPSK scheme, i.e., the case that only $A$ transmits a packet to relay. However, when $\rho=5$ (the channel gain of the link between $C$ and $B$ is much larger than that between $A$ and $B$ ), approximately the same energy is required, which imply the BER performance is mainly affected by link with lower SNR. On the other hand, the required $\left(E_{b} / N_{0}\right)_{A B}$ increases as $\rho$ decreases. Note that, when $\rho=0.2$ (the received SNR of the link between $C$ and $B$ is smaller than the link between $A$ and $B$ by $7 \mathrm{~dB}$ ), the additionally required energy for the same BER performance is approximately $7 \mathrm{~dB}$. Therefore, in this case, the BER performance is very similar to that of the case that only $C$ transmits a packet to relay. In addition, we can find that link adaptation techniques (rate selection) for PNC scheme needs to consider both links. Roughly, the transmission rate of each source node may be selected according to weak channel gain between two links because the BER performance is dominated by the weak channel both links. Roughly, the transmission rate of each source node may be selected according to weak channel gain between two links because the BER performance is dominated by the weak channel.

Fig. 4 shows the coded BER performance of the proposed PNC technique at relay node $B$ for varying $\left(E_{b} / N_{0}\right)$ and varying $\rho$ and we use a simple convolutional code as a channel coding scheme. The convolution code that used in this simulation has two memory and its minimum free distance is equal to 7 . The code rate is set to $1 / 3$ with a generator polynomial $(110,101,111)$ in binary number. As we expected in Section II, the separated decoding outperforms the direct decoding in all cases. When $\rho=1$ and we used the direct decoding instead of the separated decoding, the additionally required energy for the same BER performance is equal to $1 \mathrm{~dB}$. However, when $\rho>1$ or $\rho<1$, the performance loss of using direct decoding is negligible. For example, as we illustrated in 
Fig. 4 when $\rho=0.2$ or $\rho=5$, the performance of separated decoding and direct decoding is almost the same.

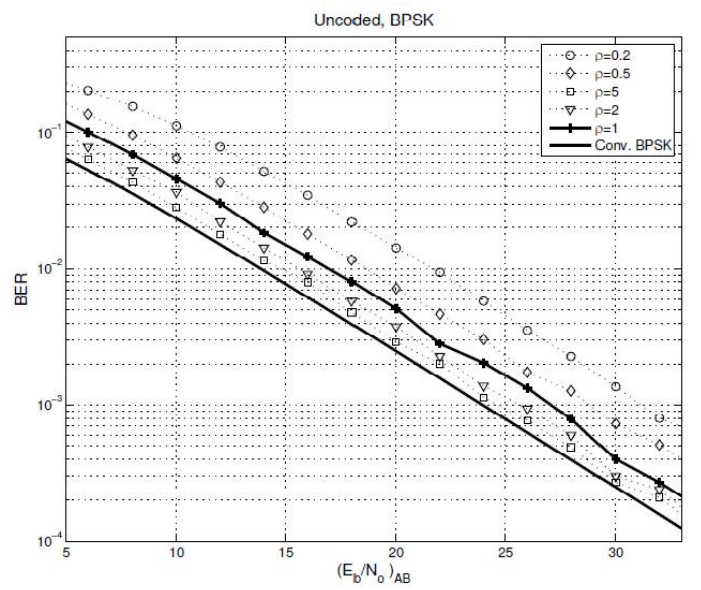

Fig. 3. BER of uncoded PNC with BPSK modulation for varying $\rho$

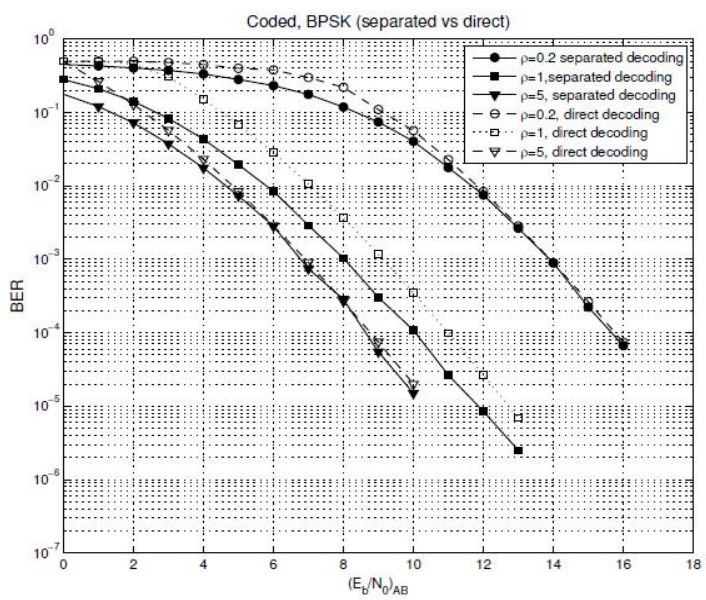

Fig. 4. BER of coded PNC with BPSK modulation for varying $\rho$

\section{CONCLUSIONS}

We proposed a practical physical layer network coding (PNC) technique without preequalization for fading channels. The proposed requires no channel state information (CSI) at both source nodes, while the conventional PNC scheme requires the full CSI of both links and strict power and phase compensation at the source nodes. We also proved that the proposed PNC technique can be easily combined with any linear channel codes. Numerical examples show that the BER performance is dominated by the weaker channel between PNC pairs and link adaptation scheme should consider the both channel gains. Finally, we find that the direct decoding can be a good solution for reducing the complexity at relay node.

\section{REFERENCES}

[1] R. Tafazolli, Technologies for the wireless future; Wireless World Research Forum (WWRF), John Wiley \& Sons, 2005.

[2] 3GPP, Radio aess (E-UTRAN); Overall description; Stage 2, 3GPP TS 36.300, V8.5.0, May 2008.

[3] 3GPP, Requirements for further advancements for E-UTRA (LTEAdvanced), 3GPP TR 36.913, V8.0.0, June 2008.

[4] IEEE, IEEE $802.16 \mathrm{~m}$ system requirements, IEEE $802.16 \mathrm{~m}-07 / 02 \mathrm{r} 4$, Oct. 2007.

[5] D. Soldani and S. Dixit, "Wireless relays for broadband access," IEEE Communi. Mag., Vol. 46, No. 3, pp. 58-66, Mar. 2008.

[6] T. M. Cover and A. El Gamal, "Capacity theorems for the relay channel," IEEE Trans. on Inform. Theory, Vol. 25, No. 5, pp. 572584, Sept. 1979.

[7] B. Rankov and A. Wittneben, "Spectral efficient protocols for halfduplex fading relay channels," IEEE Journal on Select. Areas Communi. , Vol. 25, No. 2, pp. 379-389, Feb. 2007.

[8] R. Ahlswede, N. Cai, S.-Y. R. Li, and R. W. Yeung, "Network Information Flow," IEEE Trans. Inform. Theory, Vol. 46, No. 4, pp. 1204-1216, July 2000.

[9] C. E. Shannon., "Two-way Communication Channels," In Berkeley Symp. Math. Stat. and Prob., Vol. 25, pp. 611-644, 1961.

[10] S. Katti, I. Maric, A. Goldsmith, D. Katabi, and M. Medard, "Joint relaying and network coding in wireless networks," in Proc. of IEEE Int. Symp. Inform. Theory(ISIT), June 2007.

[11] P. Larsson, N. Johansson, and K. -E. Sunell, "Coded bi-directional relaying," in Proc. of Scandinavian Workshop on Ad Hoc networks, May 2005.

[12] S. Zhang, S. Liew, and P. P.Lam, "Physical-Layer Network Coding," in Proc. of ACM MobiCom 2006, Sep. 2006.

[13] T. S. Rappaport, Wireless Communications: principles and practice, Prentice Hall, 1998.

[14] Z. Hing, K. K. Leung, D. L. Goeckel, and D. Towsley, "On the study of network coding with diversity," IEEE Trans. on Wireless Communications, Vol. 8, No. 3, pp. 1247-1259, Mar. 2009.

[15] P. Popovski and H. Yomo, "The anti-packets can increase the achievable throughput of a wireless multi-hop network," in Proc. of IEEE ICC, June 2006.

[16] P. Popovski and H. Yomo, "Physical network coding in two-way wireless relay channels," in Proc. of IEEE ICC, June 2007.

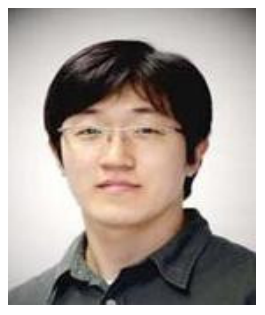

Bang Chul Jung received the B.S. degree in Electronics Engineering from Ajou University, Suwon, Korea, in 2002 and the M.S. and Ph.D degrees in Electrical \& Computer Engineering from Korea Advanced Institute of Science and Technology (KAIST), Daejeon, Korea, in 2004 and 2008, respectively. He was a research professor with KAIST Institute for Information Technology Convergence, Daejeon, Korea, until Feb. 2010. He is an assistant professor of department of Information and Communication Engineering, Gyeonsang National University, Korea. He received Bronze Prize in Intel Student Paper Contest in 2005. His research interests include orthogonal resource hopping systems for packet data transmission, OFDM systems, radio resource management, wireless scheduling algorithm, information theory, link \& system-level simulations for $4 \mathrm{G}$ wireless communication systems, adaptive modulation \& coding, and MIMO system 